\title{
MOTIVACIÓN E IMPLICACIÓN DE LOS ESTUDIANTES EN EL PROCESO DE EVALUACIÓN FORMATIVA REALIZANDO TRABAJO EN GRUPO EN
}

\section{EL AULA}

Motivation and implication of students in the process of training evaluation when performing group work in the classroom

Motivação e implicação dos alunos no processo da avaliação formativa no processo de grupo de trabalho em sala de aula

$M^{\mathrm{a}}$ de los Ángeles Castro Sastre (1)

Ana Isabel Fernández Abia (2)

Joaquín Barreiro García (3)

(1) Universidad de León, España. Teléfono:+34 987293588. Correo electrónico: macass@unileon.es

(2) Universidad de León, España. Correo electrónico: aifera@unileon.es

(3) Universidad de León, España. Correo electrónico: jbarg@unileon.es

\begin{abstract}
Resumen
El presente trabajo tiene como objetivo indagar sobre la motivación, expectativas y actitudes de los estudiantes, además de presentar una propuesta de trabajo para evaluar competencias (trabajo en grupo, comunicación oral) que en el examen final son complicadas de valorar. Es una investigación de carácter exploratorio, se aplica una encuesta inicial a los estudiantes, para conocer el punto de partida del proceso de enseñanza a la clase. Se utiliza evaluación formativa y utilizando escalas de valoración para ir evaluando el trabajo del alumno. Los resultados obtenidos permiten tanto al alumno como al profesor realizar reajustes en el proceso de aprendizaje del alumno.

Palabras clave: Motivación; Evaluación por competencias; Evaluación formativa
\end{abstract}

\begin{abstract}
The main objective of this paper is to inquire above motivation, expectations and attitudes of students, in addition to presenting a proposal of work to evaluate Motivación e implicación de los estudiantes en el proceso de evaluación formativa realizando trabajo en grupo en el aula


competencies (group work, oral communication) that in the final test are complicated to asses. The research has some exploratory character, since an initial anonymous survey is applied to the students. It uses formative evaluation and valuation scales to evaluate the work of the student. The obtained results allow both the student and the teacher to make readjustments in the learning process of the student.

Keywords: Motivation; Competency Evaluation; Formative Assessment

\section{Resumo}

Este trabalho tem como objetivo investigar a motivação, expectativas e atitudes dos alunos, além de apresentar uma proposta de trabalho para avaliar as competências (trabalho de grupo, comunicação oral) no teste final são difíceis de avaliar. Avaliação formativa é usando escalas de avaliação para estar avaliando o trabalho do aluno. Os resultados permitem tanto o aluno eo professor fazer ajustes no processo de aprendizagem do aluno.

Palavras-chave: Motivação; Competências; Avaliação Formativa

\section{Introducción}

El Espacio Europeo de Educación Superior está suponiendo un cambio en el modelo de enseñanza-aprendizaje. Estamos pasando a darle protagonismo al alumno. Por tanto, es necesario conocer la realidad del alumno en cada situación. Algunos autores como Huertas y Agudo (2003) sostienen que gran parte de los alumnos, cuando llegan a sus estudios universitarios, se suelen esforzar más y se sienten motivados cuando la materia que tiene que aprender resulta relevante, interesante y cognitivamente desafiante y si ven una aplicación práctica y útil para su vida profesional. Incluir algunas competencias en las asignaturas nos puede ayudar a conocer esta realidad en el grupo. Esto parece sencillo, pero una vez hemos incluido las competencias, surge la pregunta entre los docentes, ¿cómo evaluarlas? Una forma de evaluar las competencias es a través de la Evaluación Formativa definida por López-Pastor (2009) como: “todo proceso de evaluación cuya finalidad principal es mejorar el proceso de enseñanza-aprendizaje que tiene lugar”. Trabajar con evaluación formativa permite a los estudiantes ejercer un rol fundamental en su proceso de aprendizaje, conocer su valoración sobre aquello que han 
vivenciado, así como, darse cuenta de su rol de estudiante en cada evaluación. Por su parte, al profesor le aporta aspectos que le van a ayudar a mejorar su práctica docente y a buscar nuevas estrategias de trabajo (Gargallo et al., 2010).

En este trabajo se recoge una experiencia que se realiza en las clases de problemas, con el objetivo de ayudar a los alumnos a trabajar en grupo, así como, a desarrollar su habilidad para explicar lo que han hecho a sus compañeros.

\section{Contextualización}

Esta investigación, se está realizando en el segundo semestre del curso 16/17, con estudiantes de Segundo Grado de Ingeniería Eléctrica, concretamente en la asignatura de Tecnología de Materiales. El número de alumnos es reducido, un total de 15 alumnos. Lo que me permite tener un mayor contacto y conocimiento de los alumnos, así como, una dedicación más personalizada.

\section{Diseño y desarrollo}

El interés de este trabajo está centrado en conocer de primera mano la motivación de los alumnos hacia la asignatura de Tecnología de los Materiales, así como los conocimientos acerca de la asignatura, y el interés que tiene para ellos la misma como futuros ingenieros. Para ello se les entregó una encuesta de elaboración propia, que debían de realizar de forma anónima, con preguntas de respuesta abierta (breve) y cerrada. El objetivo con esta primera encuesta es ajustar el punto de partida del proceso de enseñanza a los estudiantes. Esto permite al profesor iniciar el proceso tal y como tenía previsto, o intentar orientarles en algunos aspectos que les haga más satisfactorio y eficaz su estudio.

El siguiente paso fue explicar a los alumnos la metodología de trabajo en las clases de problemas, a través de escalares de valoración. Estas sesiones tienen tanto carácter individual como grupal, incluyendo en ambos casos la explicación en clase de la tarea realizada. Por tanto, se les entregó otro cuestionario a los alumnos, también de elaboración propia. Este segundo cuestionario, en el que se incluían valoraciones aspectos como, implicación del alumno en la tarea, comunicación con sus compañeros, conocimientos teóricos del tema, etc.., permitió al profesor conocer la implicación de trabajo en grupo, así como, su evolución en el estudio de la materia. Además, se 
observó que ya habían trabajado algunas competencias haciendo uso de escalas de valoración en asignaturas anteriores (González-Alonso et al., 2015), lo cual facilitó la implicación del alumno en dicho trabajo, pues no era un terreno desconocido y, además, decían haber tenido muchos beneficios en su aprendizaje cuando las habían aplicado.

El trabajo en grupo consistiría en realizar los ejercicios propuestos en la clase de problemas. Para la realización de un ejercicio (grupos de 3 personas) se ejecutan las siguientes tareas: a) Analizar, Discutir y Realizar el ejercicio conjuntamente; b) Una persona voluntaria del grupo o un portavoz del mismo debía salir a explicar la resolución del ejercicio.

Con esta metodología de trabajo, además de cubrir las competencias de trabajo en grupo y comunicación oral (capacidad de explicar ideas y conceptos), el alumno también puede darse cuenta de su realidad como estudiante, de la escucha hacia el resto de compañeros, del respeto mostrado hacia las contribuciones de los demás entre otros aspectos.

\section{Evaluación y conclusiones}

Para realizar la evaluación formativa, se elaboran dos escalas de valoración, previamente conocidas por los participantes, para cada una de las fases: Fase 1.- Trabajo en grupo. Fase 2.- Explicación de tarea realizada. En la primera, vamos a conocer tanto el trabajo grupal, como el trabajo de cada integrante por separado. Al llevarse a cabo el trabajo en grupo dentro del aula, el profesor, está formando parte del grupo como observador. A la vez los alumnos también pueden ser observadores presenciales de sus propios compañeros en el momento de realizar la tarea. En la segunda escala de valoración, se evaluará la capacidad de explicación por parte de la persona al resto de compañeros, así como, su escucha ante posibles preguntas, u otras alternativas para realizar la misma tarea.

Como conclusión del trabajo realizado a día de hoy (27 de Marzo) se puede decir que les cuesta asignar tareas en un periodo corto de tiempo, planificarse y distribuir trabajo. Por otro lado, esta metodología les hace darse cuenta, de cómo llevan la planificación de estudio de la asignatura, les pone en el aquí y ahora de su situación real frente al estudio de la asignatura. Mediante esta metodología el profesor puede adelantarse a realizar reajustes en la asignatura viendo el desarrollo de los alumnos. Motivación e implicación de los estudiantes en el proceso de evaluación formativa realizando trabajo en 


\section{Referencias}

Gargallo, B., Sánchez, F. J., Ros, C., \& Ferreras, A. (2010). Estilos docentes de los profesores universitarios: la percepción de los alumnos de los buenos profesores. Revista Iberoamericana de Educación, 51(4), 1681-5653.

González-Alonso, M.I., Fernández-Díaz, R.A., Castejón Limas, M., \& Pérez-Pueyo, A. (2015). Formative assessment as a tool for acquisition of competences in engineering subjects. International Journal on Advances in Education Research (EduRe Journal), 2, 79-94

Huertas, J.A., \& Agudo, R. (2003). Concepciones de los estudiantes universitarios sobre la motivación. C. Monereo y J.I. Pozo: La universidad ante la nueva cultura educativa. Enseñar y aprender para la autonomía. Barcelona: Síntesis.

López-Pastor, V.M. (2011). El papel de la evaluación formativa en la evaluación por competencias: aportaciones de la red de evaluación formativa y compartida en docencia universitaria. Revista de Docencia Universitaria, 9(1), 159 - 173. 